Article

\title{
An Abstract Result on Projective Aggregation Functions
}

\author{
Juan C. Candeal \\ Departamento de Análisis Económico, Universidad de Zaragoza, Gran Vía 2, 50005 Zaragoza, Spain; \\ candeal@unizar.es; Tel.: +34-976-761-822
}

Received: 1 March 2018; Accepted: 19 March 2018; Published: 20 March 2018

\begin{abstract}
A general characterization result of projective aggregation functions is shown, the proof of which makes use of the celebrated Arrow's theorem, thus providing a link between aggregation functions theory and social choice theory. The result can be viewed as a generalization of a theorem obtained by Kim (1990) for real-valued aggregation functions defined on the n-dimensional Euclidean space in the context of measurement theory. In addition, two applications of the core theorem of the article are shown. The first is a simple extension of the main result to the context of multi-valued aggregation functions. The second offers a new characterization of projective bijection aggregators, thus connecting aggregation operators theory with social choice.
\end{abstract}

Keywords: aggregation functions; aggregation operators; Arrow's theorem

MSC: 39B22; 91B14; 91E45

\section{Introduction}

This short note aims to further contribute to the study of the interplay existing among aggregation functions, aggregation operators and social choice. Specifically, this paper provides a general characterization result of projective aggregation functions defined on an abstract set by using a purely social choice approach; to wit, a suitable version of Arrow's theorem (see [1]).

An aggregation process basically consists of merging pieces of information (input data) to yield some output. The information is often symbolized by means of some numerical or abstract value, and the output obtained, via the data fusion, is made by means of the so-called aggregation functions. Classical aggregation functions include those of weighted means, t-norms or t-conorms, where all the input and output variables considered are real numbers. Generalizations of the concept of an aggregation function appear when considering, for example, aggregation with ordinal and nominal scales, aggregation on posets and lattices or aggregation of preferences (see, e.g., [1-5]).

All these developments have been closely linked to an increasing number of applications to many different topics such as image processing, decision making, pattern recognition, fuzzy control and social sciences, just to mention a few of them. In addition, aggregation operators theory has particular significance in all these fields of knowledge when considering, for instance, $L$-fuzzy sets or fuzzy sets of type 2 (for details, see [6-9]; see, also, [10-12] for certain applications of aggregation operators theory to social choice).

Aggregation functions play an important role in measurement theory, as well (see [13] for a detailed account of this discipline). In this context, a key feature has to do with the preservation of particular numerical scales used to measure both the input and the output variables, which are, in turn, real variables. The concept that reflects this property is the so-called comparison meaningfulness. Built on the seminal work of Luce (1959) (see [14]), it was shown by Kim (1990) in [15] that the only real-valued aggregation functions of $n$ real variables that are continuous (continuity refers to the Euclidean topology), idempotent and comparison meaningful with respect to independent interval 
scales are the projections. This result was later generalized by other authors in several respects, noting that, in particular, continuity turned out to be an entirely redundant assumption in the previous statement. For details, see [16-21] and, more recently, [10].

In the current article, I provide a further generalization of the latter result in a much more abstract setting by studying conditions under which an aggregation function $W: X^{n} \rightarrow X$ turns out to be a projection, $X$ being an arbitrary nonempty set. Whenever $X$ has, at least, three elements, the two conditions found, that characterize such a property on $W$, are fullness and idempotency. This is the content of Theorem 2. Fullness tells us that, for a given $n$-tuple, which consists of nominal scales (a nominal scale is a bijection from $X$ onto $X$ ) in $X$, the range of $W$ over the subset of $X^{n}$ obtained by the action of the given $n$-tuple over $X$, is the whole $X$. Thus, fullness turns out to be a property about how rich the codomain of $W$ is under the action of a profile of nominal scales. Idempotency is a more familiar condition and tells us that whenever $W$ is restricted to the diagonal of $X^{n}$, then the resulting function is the identity map. The main result of the paper, namely Theorem 2, appears in Section 3. It should be noted that its proof makes use of a particular version of Arrow's theorem. This version is included in Section 2 together with some basic definitions. The fact that the cardinality of $X$ is, at least, three follows from the call to Arrow's result. In Section 4, certain consequences of Theorem 2 are shown. Firstly, a simple generalization of it is obtained in the framework of multi-valued aggregation functions, and secondly, a new characterization of projective bijection aggregators is presented. Although not developed in the article, these two consequences could be applied to the context of aggregation of preferences and aggregation of classifications, as seen, for example, in [22].

\section{Preliminaries}

Throughout the paper, the notations $n \in \mathbb{N}$ and $N:=\{1, \ldots, n\}$ will be used. Typical elements of $N$ will be denoted by $j, k, i$.

Let $X$ be a nonempty set. Vectors of length $n$ with values in $X$ will be recurrently employed. The notation $a=\left(a_{j}\right)_{j \in N}$, or simply $a=\left(a_{j}\right)$, will stand for such a typical a vector.

The set of all bijections from $X$ onto $X$ will be denoted by $B(X)$, and the symbols $\phi, \psi, \varphi$ will represent typical elements of $B(X)$. Note that, if $X$ is finite and $\phi \in B(X)$, then $\phi(X):=\{\phi(x): x \in X\}$ can be viewed as a rearrangement of $X$. The notation $|X|$ stands for the cardinal number (for details on cardinals, see, e.g., [23]) of $X$.

An aggregation function is an $X$-valued function of $n$ variables, each of them taking values in $X$; i.e., is a map $W: X^{n} \rightarrow X$. Certain properties on aggregation functions, that will be required in the main result of Section 3 are now presented.

Definition 1. An aggregation function $W: X^{n} \rightarrow X$ is said to be:

$$
\begin{aligned}
& \text { full if }\left\{W\left(\phi^{1}(x), \ldots, \phi^{n}(x)\right): x \in X\right\}=X, \text { for every } \phi^{1}, \ldots, \phi^{n} \in B(X), \\
& \text { idempotent if } W(x, \ldots, x)=x, \text { for every } x \in X, \\
& \text { projective if it is a projection, i.e., if there is } k \in N \text { such that } W(a)=a_{k} \text {, for every } a=\left(a_{j}\right) \in X^{n} .
\end{aligned}
$$

Another key and helpful concept that will be needed later is that of a bijection aggregator, or simply an aggregator. In order to define this concept, some previous notations will be useful. A profile is meant to be an element of $B(X)^{n}$. A typical profile will be denoted by $\left(\phi^{1}, \ldots, \phi^{n}\right)$. An aggregator is a map $F: B(X)^{n} \rightarrow B(X)$. I now present certain properties on bijection aggregators, some of which parallel those included in Definition 1 above for aggregation functions.

Definition 2. An aggregator $F: B(X)^{n} \rightarrow B(X)$ is, or satisfies:

(1) binary independence if for every $\left(\phi^{1}, \ldots, \phi^{n}\right),\left(\psi^{1}, \ldots, \psi^{n}\right) \in B(X)^{n}, x, y \in X$ such that $\phi^{j}(x)=$ $\psi^{j}(y)$, for all $j \in N$, it holds that $F\left(\phi^{1}, \ldots, \phi^{n}\right)(x)=F\left(\psi^{1}, \ldots, \psi^{n}\right)(y)$,

(2) unanimous if for every $\left(\phi^{1}, \ldots, \phi^{n}\right) \in B(X)^{n}, x, y \in X$ such that $\phi^{j}(x)=y$, for all $j \in N$, it holds that $F\left(\phi^{1}, \ldots, \phi^{n}\right)(x)=y$, 
projective if it is a projection, i.e., if there is $k \in N$ such that $F\left(\phi^{1}, \ldots, \phi^{n}\right)=\phi^{k}$, for every $\left(\phi^{1}, \ldots, \phi^{n}\right) \in B(X)^{n}$.

I conclude this section by showing a version of Arrow's theorem that will be employed in a decisive manner in the proof of the main result of the paper. Arrow's theorem can be considered the cornerstone of social choice theory and has strongly influenced a large number of disciplines such as logic, mathematics, economics, psychology, sociology and politics, among others. In order to prepare such a version, certain concepts need to be introduced.

A transitive and complete (hence, reflexive) binary relation $\precsim$ defined on $X$ is said to be a total preorder. A total order on $X$ is an antisymmetric total preorder. Associated with $\precsim$, the asymmetric part, also called the strict part, denoted by $\prec$, is defined as the following binary relation on $X: x \prec y$ if and only if $x \precsim y$ and $\neg(y \precsim x$ ) (or, equivalently, since $\precsim$ is a total preorder, $x \prec y$ if and only if $\neg(y \precsim x))$. Note that the asymmetric part of a total order defined on $X$ turns out to be a linear order on $X$. Similarly, its symmetric part, denoted by $\sim$, is defined by: $x \sim y$ if and only if $x \precsim y$ and $y \precsim x$. The symmetric part of a total preorder turns out to be an equivalence relation on $X$. The binary relation defined on quotient set $X / \sim$ as: $[x] \precsim[y] \Leftrightarrow x \precsim y,(x, y \in X)$, is a total order on it (In the economics literature, a total preorder is also referred to as a preference and as a social welfare ordering in social choice theory. In addition, the asymmetric part and the symmetric part of a total preorder are called in economics the strict preference and the indifference relation, respectively. The set $X$ is usually termed in these fields as the choice set, or the set of alternatives, or the set of social outcomes, and $N$ stands for the individuals in the society.). A well ordering, or an ordinal, on $X$ is a total order with the additional property that each nonempty subset of $X$ has a first element.

Denote by $\mathcal{R}, \mathcal{T}$ and $\mathcal{W}$ the set of all total preorders, total orders and well orderings, respectively, defined on $X$. Obviously, $\mathcal{W} \subseteq \mathcal{T} \subseteq \mathcal{R}$.

Following Arrow (see [1]), a social welfare function turns out to be a map $G: \mathcal{R}^{n} \rightarrow \mathcal{R}$. Each $n$-tuple $\left(\precsim^{1}, \ldots, \precsim^{n}\right) \in \mathcal{R}^{n}$ is said to be a profile of individual preferences. The interpretation is that $G$ maps profiles of individual preferences into social preferences. For each element of $G\left(\mathcal{R}^{n}\right)$, say $G\left(\precsim^{1}, \ldots, \precsim^{n}\right)$, the somewhat unpleasant notation $\precsim_{G\left(\precsim^{1}, \ldots, \precsim^{n}\right)}$ and $\prec_{G\left(\precsim^{1}, \ldots, \precsim^{n}\right)}$ for its asymmetric part, will be used.

Definition 3. A social welfare function $G$ satisfies:

(i) the condition of independence of irrelevant alternatives if for any two profiles of individuals preferences $\left(\precsim_{1}, \ldots, \precsim_{1}^{n}\right),\left(\precsim_{2}, \ldots, \precsim_{2}^{n}\right) \in \mathcal{R}^{n}$ and any two alternatives $x, y \in X$ such that $x \precsim_{1}^{j} y \Leftrightarrow x \precsim_{2}^{j}$ $y$, and $y \precsim_{1}^{j} x \Leftrightarrow y \precsim_{2}^{j} x$, for all $j \in N$, it holds that $x \precsim_{G\left(\precsim_{1}^{1}, \ldots, \precsim_{1}^{n}\right)} y \Leftrightarrow x \precsim_{G\left(\precsim_{2}, \ldots, \precsim_{2}\right)} y$ and $y \precsim_{G\left(\precsim_{1}, \ldots, \precsim n\right)} x \Leftrightarrow y \precsim_{G\left(\precsim_{2}^{1}, \ldots, \precsim 2 n\right)} x$,

(ii) the Pareto condition if for any profile $\left(\precsim^{1}, \ldots, \precsim^{n}\right) \in \mathcal{R}^{n}$ and any pair of alternatives $x, y \in X$ such that $x \prec^{j} y$, for all $j \in N$, it holds that $x \prec_{G\left(\precsim^{1}, \ldots, \precsim^{n}\right)} y$.

Theorem 1. Suppose that the cardinality of $X$ is, at least, three. Let $G: \mathcal{R}^{n} \rightarrow \mathcal{R}$ be a social welfare function that satisfies the condition of independence of irrelevant alternatives and the Pareto condition. Then, there is $k \in N$ such that for any profile $\left(\precsim^{1}, \ldots, \precsim^{n}\right) \in \mathcal{R}^{n}$ and any pair of alternatives $x, y \in X$, it holds that $x \prec^{k} y$ entails $x \prec_{G\left(\precsim^{1}, \ldots, \precsim n\right)} y[1]$.

Arrow's statement can be reinforced provided that certain qualifications on the domain of the social welfare function $G$ are done while maintaining the meaning of both conditions that appear in Definition 3 and the fact that $|X| \geq 3$. Let $\mathcal{S} \subseteq \mathcal{R}$. If the domain of $G$ is restricted to $\mathcal{S}^{n}$, then the resulting map is called a partial social welfare function. A partial social welfare function $G: \mathcal{S}^{n} \rightarrow \mathcal{R}$ is said to be projective whenever there is $k \in N$ such that $G\left(\precsim^{1}, \ldots, \precsim^{n}\right)=\precsim^{k}$, for every $\left(\precsim^{1}, \ldots, \precsim^{n}\right) \in \mathcal{S}^{n}$. In the social choice literature, a projective partial social welfare function is usually referred to as a strong dictatorship, and individual $k$ is called a strong dictator. 
I now comment on two important cases where a partial social welfare function turns out to be projective. In both situations, the proof is a direct consequence of the original proof of Arrow's theorem (see [1] or [24] for an alternative view). The first one is $\mathcal{S}=\mathcal{T}$. The second one, which actually is the variant I will use later, involves ordinals. Let $\leq \in \mathcal{W}$ be a well ordering defined on $X$ which does exist by Zorn's lemma. Let $\phi \in B(X)$. Consider the binary relation, denoted by $\phi \leq$, on $X$ defined by: $x \phi \leq y \Leftrightarrow \phi(x) \leq \phi(y),(x, y \in X)$. Clearly, $\phi_{\leq} \in \mathcal{W}$. Let $\mathcal{W}_{\leq}$stand for the following subset of $\mathcal{W}, \mathcal{W}_{\leq}:=\left\{\phi_{\leq}: \phi \in B(X)\right\}$. Let $\mathcal{S}=\mathcal{W}_{\leq}$. Then, a partial social welfare function $G: \mathcal{S}^{n} \rightarrow \mathcal{R}$ that satisfies the condition of independence of irrelevant alternatives and the Pareto condition is projective.

\section{The Main Result}

In this section, the statement of the major result of the paper is presented. As already said in the Introduction, it turns out to be a characterization of projective aggregation functions on an abstract set, which contains, at least, three elements. In addition, certain variants of this result are also included, which require slight modifications of the concept of being full.

Theorem 2. Let $|X| \geq 3$. Then, for an aggregation function $W: X^{n} \rightarrow X$, the following conditions are equivalent:

(i) W is projective.

(ii) W is idempotent and full.

Proof. That (i) entails (ii) is obvious, so I will focus on the other implication, which will be developed in four steps.

Step 1. For each $a=\left(a_{j}\right) \in X^{n}, W(a) \in\left\{a_{1}, \ldots, a_{n}\right\}$. Indeed, take the elements of $\left\{a_{1}, \ldots, a_{n}\right\}$ avoiding repetitions. Therefore, without loss of generality, it may be assumed that $a_{i} \neq a_{j}$, for all $i \neq j$. Let $A:=\left\{a_{1}, \ldots, a_{n}\right\}$. Then, for each $j \in N$, consider the function $\phi^{j}: X \rightarrow X$ defined by: $\phi^{j}\left(a_{i}\right)=a_{i+j-1(\text { modulo } n)}$ and $\phi^{j}(x)=x$, for all $x \in X \backslash A$ (In this definition, I use the convention $a_{0}=a_{n}$. Note that each $\phi^{j}$ maps the set $\left\{a_{1}, \ldots, a_{n}\right\}$ into itself in a bijective way starting from $a_{j}$ following the usual order in $N$. In addition, observe that if there was any repetition in $\left\{a_{1}, \ldots, a_{n}\right\}$, say $a_{k}=a_{l}$, with $k \neq l$, then $\phi^{k}=\phi^{l}$.). Clearly, $\phi^{j} \in B(X)$, for every $j \in N$.

Now, because $W$ is idempotent, it holds that $\left\{W\left(\phi^{1}(x), \ldots, \phi^{n}(x)\right): x \in X \backslash A\right\}=X \backslash A$. Hence, since $W$ is full, $\left\{W\left(\phi^{1}(x), \ldots, \phi^{n}(x)\right): x \in A\right\}=A$. Thus, for $x=a_{1}$, it holds that $W(a)=W\left(a_{1}, \ldots, a_{n}\right)=W\left(\phi^{1}\left(a_{1}\right), \ldots, \phi^{n}\left(a_{1}\right)\right) \in\left\{a_{1}, \ldots, a_{n}\right\}$, which proves Step 1.

Step 2. Let there be given $a=\left(a_{j}\right), b=\left(b_{j}\right) \in X^{n}$ such that $a_{j} \neq b_{j}$, for all $j \in N$. Then, $W(a) \neq W(b)$. In order to see this, first take the elements of $\left\{a_{1}, \ldots, a_{n}\right\}$ and $\left\{b_{1}, \ldots, b_{n}\right\}$, avoiding repetitions. Therefore, as above, it may be assumed that $a_{i} \neq a_{j}$ and also that $b_{i} \neq b_{j}$, for all $i \neq j$. Let $A:=\left\{a_{1}, \ldots, a_{n}\right\}, B:=\left\{b_{1}, \ldots, b_{n}\right\}$ and $C:=A \cup B$. Then, for each $j \in N$, consider a function $\phi^{j}: X \rightarrow X$ accomplishing the following requirements: $\phi^{j}$ is a bijection from $C$ onto $C$ such that $\phi^{j}\left(a_{1}\right)=a_{j}, \phi^{j}\left(b_{1}\right)=b_{j}$ and $\phi^{j}(x)=x$, for all $x \in X \backslash C$. Note that such a function can be easily built and also that $\phi^{j} \in B(X)$, for every $j \in N$.

Thus, since $W$ is idempotent, it holds that $\left\{W\left(\phi^{1}(x), \ldots, \phi^{n}(x)\right): x \in X \backslash C\right\}=X \backslash C$. Hence, since $W$ is full, $\left\{W\left(\phi^{1}(x), \ldots, \phi^{n}(x)\right): x \in C\right\}=C$. Note that, for $x=a_{1} \in C$, $W(a)=W\left(a_{1}, \ldots, a_{n}\right)=W\left(\phi^{1}\left(a_{1}\right), \ldots, \phi^{n}\left(a_{1}\right)\right)$ and for $x=b_{1} \in C, W(b)=W\left(b_{1}, \ldots, b_{n}\right)=$ $W\left(\phi^{1}\left(b_{1}\right), \ldots, \phi^{n}\left(b_{1}\right)\right)$. Thus, necessarily, $W(a) \neq W(b)$.

Step 3. Let $\leq \in \mathcal{W}$ be a (fixed) well ordering on $X$. The inequalities " $<$ " and " $>$ ", between elements of $X$, have the usual meanings. I now prove that $W$ is strongly monotone in the following sense: for any $a=\left(a_{j}\right), b=\left(b_{j}\right) \in X^{n}$ such that $a_{j}<b_{j}$, for all $j \in N$, it holds that $W(a)<W(b)$. Suppose, by way of contradiction, that there are $a=\left(a_{j}\right), b=\left(b_{j}\right) \in X^{n}$ such that $a_{j}<b_{j}$, for all $j \in N$ and $W(a)>W(b)$ (note that the situation $W(a)=W(b)$ violates the condition of $W$ being full as seen in Step 2 above). Let $a_{k}, a_{l}$ and $b_{m}$ be defined as follows: $a_{k}=\min _{j \in N} a_{j}, a_{l}=W(a)$ 
and $b_{m}=W(b)$. Note that the latter two numbers exist by Step 1, and also, it holds that $a_{k}<b_{m}<a_{l}$. Define $\alpha=\left(\alpha_{j}\right), \beta=\left(\beta_{j}\right) \in X^{n}$ in the following way:

$$
\alpha_{j}=\left\{\begin{array}{cc}
a_{l} & \text { if } j \in A \\
a_{k} & \text { otherwise }
\end{array}, \quad \beta_{j}=\left\{\begin{array}{cc}
a_{l} & \text { if } j \in B \\
b_{m} & \text { otherwise }
\end{array}\right.\right.
$$

where $A:=\left\{j \in N: a_{j}=a_{l}\right\}$ and $B:=\left\{j \in N: b_{j}=b_{m}\right\}$. Note that $\alpha_{j} \neq \beta_{j}$, for all $j$. Thus, by Step 2 , it holds that $W(\alpha) \neq W(\beta)$. Now, a contradiction will be reached by showing that $W(\alpha)=W(\beta)$. In order to see this, define $\gamma=\left(\gamma_{j}\right) \in X^{n}$ as follows: $\gamma_{j}=\left\{\begin{array}{cc}a_{k} & \text { if } j \in A \\ a_{l} & \text { otherwise }\end{array}\right.$. Then, because $W(a)=a_{l}, W(\gamma) \in\left\{a_{k}, a_{l}\right\}$ and $\gamma_{j} \neq a_{j}$, for all $j$, it holds that $W(\gamma)=a_{k}$. Similarly, $W(\alpha) \in\left\{a_{l}, a_{k}\right\}$ and $\gamma_{j} \neq \alpha_{j}$, for all $j$. Hence, $W(\alpha)=a_{l}$. With a similar argument, by using now $W(b)=b_{m}$, $W(\beta) \in\left\{a_{l}, b_{m}\right\}$ and $\beta_{j} \neq b_{j}$, for all $j$, it holds that $W(\beta)=a_{l}$, which gives the desired contradiction.

Step 4. To finish the proof, consider the aggregator $F: B(X)^{n} \rightarrow B(X)$ defined as follows $F\left(\phi^{1}, \ldots, \phi^{n}\right)(x)=W\left(\phi^{1}(x), \ldots, \phi^{n}(x)\right)$, for every $\left(\phi^{1}, \ldots, \phi^{n}\right) \in B(X)^{n}, x \in X$. By Step 2 , joint to the fact that $W$ is full, it follows that $F\left(B(X)^{n}\right) \subseteq B(X)$. I now prove that $F$ satisfies binary independence. In order to see this, let $\left(\varphi^{1}, \ldots, \varphi^{n}\right),\left(\psi^{1}, \ldots, \psi^{n}\right) \in B(X)^{n}$ such that there are $x, y \in X$ for which $\varphi^{j}(x)=\psi^{j}(y)$, for all $j \in N$. Then, $F\left(\varphi^{1}, \ldots, \varphi^{n}\right)(x)=W\left(\varphi^{1}(x), \ldots, \varphi^{n}(x)\right)=W\left(\psi^{1}(y), \ldots, \psi^{n}(y)\right)=$ $F\left(\psi^{1}, \ldots, \psi^{n}\right)(y)$, and so, $F$ satisfies binary independence. Moreover, since by Step 3 above, $W$ is strongly monotone, $F$ satisfies the following monotonicity property: for every $\left(\phi^{1}, \ldots, \phi^{n}\right) \in B(X)^{n}$, $x, y \in X$ such that $\phi^{j}(x)<\phi^{j}(y)$, for all $j \in N$, it holds $F\left(\phi^{1}, \ldots, \phi^{n}\right)(x)<F\left(\phi^{1}, \ldots, \phi^{n}\right)(y)$.

Now, consider the set $\mathcal{S}=\mathcal{W}_{\leq}$introduced in the last paragraph of Section 2. Define the partial social welfare function $G: \mathcal{S}^{n} \rightarrow \mathcal{R}$ as follows: $G\left(\phi_{\leq}^{1}, \ldots, \phi_{\leq}^{n}\right):=F\left(\phi^{1}, \ldots, \phi^{n}\right)_{\leq}$, for every $\left(\phi_{\leq}^{1}, \ldots, \phi_{\leq}^{n}\right) \in \mathcal{S}^{n}$. The two properties of $F$ proven in the above paragraph clearly entail that $G$ satisfies both the Pareto condition and the condition of independence of irrelevant alternatives. Therefore, by the second variant of Arrow's theorem mentioned at the end of Section 2, there is $k \in N$ such that $G\left(\phi_{\leq}^{1}, \ldots, \phi_{\leq}^{n}\right)=\phi_{\leq}^{k}$, for every $\left(\phi_{\leq}^{1}, \ldots, \phi_{\leq}^{n}\right) \in \mathcal{S}^{n}$. However, then, it clearly holds that $F\left(\phi^{1}, \ldots, \phi^{n}\right)=\bar{\phi}^{k}$, for every $\left(\phi^{1}, \ldots, \phi^{n}\right) \in B(X)^{n}$. Hence, $W(a)=a_{k}$, for every $a=\left(a_{j}\right) \in X^{n}$, and the proof is finished.

The next two examples show that fullness and idempotency are independent conditions.

Example 1. (i) Let $X=\mathbb{R}$, and define $W: \mathbb{R}^{2} \rightarrow \mathbb{R}$ as follows: $W(x, y)=\max \{x, y\}$. Obviously, $W$ is a non-projective and idempotent aggregation function. However, it is not full since by taking the two bijections $\phi(x)=x$ and $\psi(x)=-x$, it holds that $\{W(\phi(x), \psi(x)): x \in \mathbb{R}\}=[0, \infty) \subsetneq \mathbb{R}$.

(ii) Let $X=\mathbb{R}$, and define $W: \mathbb{R}^{2} \rightarrow \mathbb{R}$ as follows: $W(x, y)=x^{3}$. Obviously, $W$ is a non-projective and full aggregation function, which fails to be idempotent.

Remark 1. (i) The assumption $|X| \geq 3$ cannot be ruled out from the statement of Theorem 2. Indeed, take $X=\{x, y\}$. Define $W: X^{3} \rightarrow X$ as follows: $W(x, x, x)=x, W(y, y, y)=y$, $W(x, x, y)=x, W(x, y, x)=x, W(y, x, x)=x, W(y, y, x)=y, W(y, x, y)=y, W(x, y, y)=y$. Clearly, $W$ so-defined is idempotent and full. However, it is not projective.

(ii) Alternative characterizations of projective functions can be shown provided that the concept of fullness is suitably modified. For instance, suppose that $Z$ is a nonempty set such that $|Z|=|X|$, and denote by $B(Z, X)$ the set of all bijections from $Z$ onto $X$. An aggregation function $W: X^{n} \rightarrow X$ is said to be full with respect to bijections if $\left\{W\left(b^{1}(z), \ldots, b^{n}(z)\right): z \in Z\right\}=X$, for every $b^{1}, \ldots, b^{n} \in B(Z, X)$. Then, following the proof of Theorem 2 with the obvious modifications, the next result is in order: assume $|X| \geq 3$. Then, $W$ is projective iff it is idempotent and full with respect to bijections.

(iii) In relation to Remark 1 (ii) above, suppose now that $|Z| \geq|X|$. Let $S(Z, X)$ stand for the set of all surjections from $Z$ onto $X$. An aggregation function $W: X^{n} \rightarrow X$ is said to be full with respect to surjections if $\left\{W\left(s^{1}(z), \ldots, s^{n}(z)\right): z \in Z\right\}=X$, for every $s^{1}, \ldots, s^{n} \in S(Z, X)$. Translating into 
this context the arguments used in the proof of Theorem 2, the following result can be proven: assume $|X| \geq 3$. Then, $W$ is projective iff it is idempotent and full with respect to surjections (This result requires consideration of a well ordering, say $\leq$, on $X$. Then, for every $s \in S(Z, X)$, define the binary relation, denoted by $s_{\leq}$, on $Z$ as follows: $z s_{\leq} t \Leftrightarrow s(z) \leq s(t),(z, t \in Z)$. Consider the following subset of binary relations on $Z, \mathcal{W}_{\leq}:=\left\{s_{\leq}: s \in S(Z, X)\right\}$. Note that, for each $s \in S(Z, X), s_{\leq}$is a total preorder on $Z$ that induces a well ordering on the quotient set $Z / \sim_{s_{\leq}}$. Then, the variant of Arrow's theorem used in this situation states that a partial social welfare function $G:\left(\mathcal{W}_{\leq}\right)^{n} \rightarrow \mathcal{R}$ that satisfies the condition of independence of irrelevant alternatives and the Pareto condition is projective.). A similar result can be given by adapting the fullness condition to the context of injections from $Z$ into $X$ provided that $|Z| \leq|X|$.

\section{Some Consequences of the Main Result}

I conclude the paper with two applications of Theorem 2. The first one is a simple generalization of this result to the context of multi-valued aggregation functions. Let $X$ be a nonempty set and $n \in \mathbb{N}$. Denote by $\mathcal{P}(X)=2^{X} \backslash \varnothing$. Then, a multi-valued aggregation function is a map $G: X^{n} \rightarrow \mathcal{P}(X)$. A multi-valued aggregation function $G$ is projective if there is $k \in\{1, \ldots, n\}$ such that $a_{k} \in G(a)$, for every $a=\left(a_{j}\right) \in X^{n}$. An aggregation function $V: X^{n} \rightarrow X$ is said to be a selection of $G$ if $V(a) \in G(a)$, for every $a=\left(a_{j}\right) \in X^{n}$.

The next result, which is an obvious consequence of Theorem 2, is now in order.

Corollary 1. Let $|X| \geq 3$. Then, for a multi-valued aggregation function $G: X^{n} \rightarrow \mathcal{P}(X)$, the following conditions are equivalent:

(i) G is projective.

(ii) G admits a selection that is idempotent and full.

As the second application of Theorem 2, the following characterization of a (bijection) aggregator is shown.

Corollary 2. Let $|X| \geq 3$. Then, for an aggregator $F: B(X)^{n} \rightarrow B(X)$, the following conditions are equivalent:

(i) $F$ is projective.

(ii) $\quad F$ is unanimous and satisfies binary independence.

Proof. That (i) implies (ii) is obvious. In order to see the converse, assume that $F: B(X)^{n} \rightarrow B(X)$ is a unanimous aggregator that satisfies binary independence. Define the aggregation function $W: X^{n} \rightarrow X$ as follows: for each $a=\left(a_{j}\right) \in X^{n}, W(a)=F\left(\phi^{1}, \ldots, \phi^{n}\right)(x)$, where $\left(\phi^{1}, \ldots, \phi^{n}\right) \in B(X)^{n}$, $x \in X$ are such that $\phi^{j}(x)=a_{j}$, for all $j \in N$. Since $F$ satisfies binary independence, $W$ is well defined (i.e., the value $F\left(\phi^{1}, \ldots, \phi^{n}\right)(x)$ does not depend on the particular $\left(\phi^{1}, \ldots, \phi^{n}\right) \in B(X)^{n}$ and $x \in X$ chosen). In addition, the unanimity of $F$ entails that $W$ is idempotent. Moreover, since the image of $F$ lies on $B(X)$, it clearly follows that $W$ is full. Hence, by Theorem $2, W$ is projective, and so, $F$ is.

Remark 2. (i) It should be noted that, according to Remarks 1(ii) and 1(iii), alternative versions of Corollary 2 can be established by replacing $B(X)$ with $B(Z, X)$ and $S(Z, X)$, respectively.

(ii) Corollary 2 can be given an interpretation in the fuzzy framework. Assume $U$ is a finite universe, with states $\left\{u_{1}, \ldots, u_{m}\right\}$, and denote by $X$ a set of fuzzy numbers defined on $U$ such that $|X| \geq 3$ (Note that each number in $X$ can be identified with a vector in the m-dimensional unit cube where, for every $l \in\{1, \ldots, m\}$, the l-component might be interpreted as the uncertainty, or vagueness, that state $u_{l}$ occurs. Then, $X$ could be thought of as the set of possible uncertain situations to be managed.). Then, $B(X)$ represents the set of all possible rearrangements of the referenced uncertain situations. Further, suppose that there are $n$ individuals, $n \in \mathbb{N}$, each of them presenting a particular arrangement of the uncertain situations, and a final arrangement needs to be reached as an aggregation procedure 
of the individual proposals. Then, Corollary 2 provides necessary and sufficient conditions for such an aggregation procedure to coincide with the proposal of some individual.

\section{Conclusions}

In this paper, a very general characterization result concerning projective aggregation functions is presented. This result is motivated by a theorem obtained by Kim (1990), for real-valued aggregation functions defined on the $n$-dimensional Euclidean space, in the measurement theory context. A key feature of the approach followed in the paper is that the proof of the core result is based on a particular version of Arrow's theorem, thus providing a link between aggregation functions theory and social choice theory. Some extensions and consequences are presented, as well; in particular, a generalization of the main result to the framework of multi-valued aggregation functions and a characterization of projective bijection aggregators. Although not developed in the article, it is pointed out that these consequences could have certain applications to the environment of the aggregation of preferences and aggregation of classifications.

Acknowledgments: This work has been partially supported by the Research Project ECO2012-34828 (Spain). I am grateful to Esteban Induráin, who suggested that I illustrate Corollary 2 with an example coming from the "fuzzy world". I also thank two referees for providing me with valuable comments that have led to an improved version of the manuscript. Technical support of the Assistant Editor L. Shen is also acknowledged.

Conflicts of Interest: The author declares no conflict of interest.

\section{References}

1. Arrow, K.J. Social Choice and Individual Values, 2nd ed.; Wiley: New York, NY, USA, 1963.

2. Zadeh, L.A. Fuzzy sets. Inf. Control 1965, 8, 338-353.

3. Zadeh, L.A. Quantitative fuzzy semantics. Inf. Sci. 1971, 3, 159-176.

4. Dubois, D.; Prade, H. Fuzzy Sets and Systems: Theory and Applications; Academic Press: New York, NY, USA, 1980.

5. Goguen, J.A. L-fuzzy sets. J. Math. Anal. Appl. 1967, 18, 145-174.

6. Aczél, J. A Short Course on Functional Equations; Kluwer: Alphen aan den Rijn, The Netherlands, 1987.

7. Calvo, T.; Mayor, G.; Mesiar, R. (Eds.) Aggregation Operators: New Trends and Applications; Springer: Berlin, Germany, 2002.

8. Bustince, H.; Fernández, J.; Mesiar, R.; Calvo, T. (Eds.) Aggregation Functions in Theory and in Practise; Springer: Berlin, Germany, 2002.

9. Tizhoosh, H. Image thresholding using type II fuzzy sets. Pattern Recognit. 2005, 38, 2363-2372.

10. Candeal, J.C. Aggregation operators, comparison meaningfulness and social choice. J. Math. Psychol. 2016, $75,19-25$.

11. De Miguel, L.; Campión, M.J.; Candeal, J.C.; Induráin, E.; Paternain, D. Pointwise aggregation of maps: Its structural functional equation and some applications to social choice. Fuzzy Sets Syst. 2017, 325, 137-151.

12. Candeal, J.C.; Induráin, E. Point-sensitive aggregation operators: Functional equations and applications to social choice. Int. J. Uncertain. Fuzziness 2017, 25, 973-986.

13. Krantz, D.; Luce, R.D.; Suppes, P.; Tversky, A. Foundations of Measurement. Vol. I: Additive and Polynomial Representations; Academic Press: New York, NY, USA, 1971.

14. Luce, R.D. On the possible psychophysical laws. Psychol. Rev. 1959, 66, 81-95.

15. Kim, S.-R. On the possible scientific laws. Math. Soc. Sci. 1990, 20, 19-36.

16. Marichal, J.L.; Mathonet, P. On comparison meaningfulness of aggregation functions. J. Math. Psychol. 2001, 45, 213-223.

17. Marichal, J.L. On order invariant synthesizing functions. J. Math. Psychol. 2002, 46, 661-676.

18. Marichal, J.L.; Mesiar, R.; Rückschlossová, A. A complete description of comparison meaningful functions. Aequ. Math. 2005, 69, 309-320.

19. Grabisch, M.; Marichal, J.L.; Mesiar, R.; Pap, E. Aggregation Functions; Encyclopedia of Mathematics and Its Applications 127; Cambridge University Press: Cambridge, UK, 2009. 
20. Marichal, J.L.; Mesiar, R. A complete description of comparison meaningful aggregation functions mapping ordinal scales into an ordinal scale: A state of the art. Aequ. Math. 2009, 77, 207-236.

21. Aczél, J.; Roberts, F.S. On the possible merging functions. Math. Soc. Sci. 1989, 17, 205-243.

22. Maniquet, F.; Mongin, P. A theorem on aggregating classifications. Math. Soc. Sci. 2016, 79, 6-10.

23. Dugundji, J. Topology; Allyn and Bacon: Boston, MA, USA, 1966.

24. Campbell, J.C.; Kelly, J.R. Impossibility theorems in the Arrovian framework. In Handbook of Social Choice and Welfare; Arrow, K.J., Sen, A., Suzumura, K., Eds.; Elsevier: Amsterdam, The Netherlands, 2002; Volume 1, pp. 35-94.

(C) 2018 by the author. Licensee MDPI, Basel, Switzerland. This article is an open access article distributed under the terms and conditions of the Creative Commons Attribution (CC BY) license (http://creativecommons.org/licenses/by/4.0/). 